\title{
Determining the water damage resistance of nano-clay modified bitumens using the indirect tensile strength and surface free energy methods
}

\begin{abstract}
Water infiltration into asphalt mixtures is one the serious distresses which lowers the performance of the top layer of road pavements. This problem is even worse in tropical countries which experience high temperature and heavy rainfalls. Therefore, this study aims to evaluate the effects of water infiltration on a $60 / 70$ penetration grade bitumen vis-a-vis bitumen modified with 2 and 4\% nano-clay. The Fourier Transform InfraRed (FTIR) test was done to identify any chemical changes that might occur in the nano-clay and the bitumen. The loose and compacted mixtures of the nano-clay modified bitumen were tested to determine their resistance to water damage. The loose mixture was tested by conducting the boiling water, the Vialit adhesion, and surface free energy (SFE) tests, while the compacted mixture was tested using the indirect tensile strength (ITS) test. Aging simulation was done for samples tested using SFE and ITS. The results of the tests show that adding nano-clay alters the mixtures' ability to resist water damage. The improvement in the samples varies depending on the type of binder as well as its aging stage. Unaged bitumen modified with $2 \%$ nano-clay show better ability to resist water damage compared to both the unmodified bitumen and the bitumen modified with 4\% nano-clay. However, after being subjected to short-term aging (STA) and long-term aging (LTA), the results of both the ITS and SFE tests show that the addition of $4 \%$ nano-clay enhance the bitumen's resistance to water damage in comparison with the unmodified bitumen and the bitumen modified with $2 \%$ nano-clay. The addition of nano-clay reduces water's effect on the binder, hence indicating the benefit of using nano-clay as an additive to mitigate water effect.
\end{abstract}

Keyword: Bitumen; Nano-clay; Water damage; Indirect tensile strength; Surface free energy 